\title{
Effect of low-intensity focused ultrasound on endothelin-1, nitrogen monoxide and oxytocin receptor in the uterine tissues of Sprague-Dawley rats following abortion
}

\author{
YANXIA ZHANG, JUFANG GUO, CHUAN LIN, LU LU and CHENGZHI LI \\ State Key Laboratory of Ultrasound Engineering in Medicine Co-founded by Chongqing and the Ministry of Science \\ and Technology, Chongqing Key Laboratory of Ultrasound in Medicine and Engineering, \\ College of Biomedical Engineering, Chongqing Medical University, Chongqing 400016, P.R. China
}

Received August 3, 2015; Accepted November 11, 2015

DOI: $10.3892 /$ br.2016.581

\begin{abstract}
The aim of the present study was to investigate the effect of low-intensity focused ultrasound on endothelin-1 (ET-1), nitrogen monoxide (NO) and oxytocin receptor (OXTR) levels in the uterine tissues of Sprague-Dawley (SD) rats following abortion. A total of $30 \mathrm{SD}$ rats undergoing complete abortion were randomly divided into ultrasound irradiation and sham irradiation groups (15 rats per group). The rats in the ultrasound irradiation group were treated with low-intensity ultrasound (sound intensity, $2 \mathrm{~W} / \mathrm{cm}^{2}$; frequency, $0.8 \mathrm{MHz}$ ) for $30 \mathrm{~min}$ daily for 5 consecutive days, and those in the sham irradiation group received sham treatment. The uterine tissue was removed to measure the levels of ET-1, NO and OXTR using the enzyme-linked immunosorbent assay and immunohistochemistry, respectively. The ET-1 level in the uterine tissues was significantly higher in the ultrasound irradiation group compared to the sham irradiation group $(\mathrm{P}<0.05)$; however, the NO level was similar in the 2 groups $(\mathrm{P}>0.05)$. In the uterine myometrium and endometrium, the strong positive expression of OXTR was observed in the ultrasound irradiation group, which was significantly higher compared to the sham irradiation group $(\mathrm{P}<0.05)$. Low-intensity ultrasound could promote uterine involution by increasing ET-1 levels, modifying the balance of ET-1 and NO, and enhancing the expression of OXTR in the uterine myometrium and endometrium.
\end{abstract}

Correspondence to: Professor Chengzhi Li, State Key Laboratory of Ultrasound Engineering in Medicine Co-founded by Chongqing and the Ministry of Science and Technology, Chongqing Key Laboratory of Ultrasound in Medicine and Engineering, College of Biomedical Engineering, Chongqing Medical University, 1 Medical College Road, Chongqing 400016, P.R. China

E-mail: lichengzhi@cqmu.edu.cn

Key words: low-intensity focused ultrasound, uterine involution, endothelin-1, nitric oxide, oxytocin receptor

\section{Introduction}

Low-intensity focused ultrasound penetrates the topical tissues or organs as a low-dose mechanical energy, and is widely used in the treatment of musculoskeletal injury (1) and uterine involution. While the role of low-intensity focused ultrasound in postpartum uterine involution has attracted increasing attention and recognition, its mechanism in promoting uterine smooth muscle contraction remains to be elucidated. A randomized controlled study was performed to examine the clinical application of low-intensity focused ultrasound in uterine involution by observing its effect on the levels of endothelin-1 (ET-1), nitrogen monoxide (NO) and oxytocin receptor (OXTR) in uterine tissues of Sprague-Dawley (SD) rats following abortion.

\section{Materials and methods}

Animals. A total of 60 healthy SD rats $(55$ females and 5 males), aged $\leq 3$ months (nulliparous females, 180-250 g; males, 250-350 g) were maintained in individually ventilated cages in the Animal Center of Chongqing Medical University (Chongqing, China) (room temperature, $18-25^{\circ} \mathrm{C}$; humidity, 40-60\%; noise, $\leq 60 \mathrm{~dB}$ ). The animal research protocol was approved by the Animal Care and Use Committee of Chongqing Medical University.

Drugs and reagents. Mifepristone tablets configured in a liquid with a concentration of $1.66 \mathrm{mg} / \mathrm{ml}$ and $0.01 \mathrm{~g} / \mathrm{ml}$ (approval numbers: Zhunzi H20033551 and H20073696, respectively) were purchased from Gedian Humanwell Hubei Pharmaceutical Co., Ltd. (Hubei, China). The ET-1 enzyme-linked immunosorbent assay (ELISA) detection kit was from Nanjing Jiancheng Technology Co., Ltd. (Nanjing, China). The rabbit anti-mouse OXTR antibody (ab101617) was purchased from Abcam (Cambridge, MA, USA) and the streptavidin-horseradish peroxidase rabbit secondary antibody kit was from KangWei Century Technology Co., Ltd. (Beijing, China).

Instruments. The ultrasound therapeutic device for involution of the uterus (CKC100) was purchased from Chongqing 
Haifu Medical Technology Co., Ltd. (Chongqing, China), the microplate reader (352) was from LabSystems Multiskan MS (Helsinki, Finland), the washer (ACB) was purchased from Thermo LabSystems (Helsinki, Finland), the micro-speed centrifuge (TG16W) was from Changsha Pingfan Instrument and Meter Co., Ltd. (Changsha, China), the water-incubator (GNP-9080) was from Shanghai Wujiu Automation Equipment, Co., Ltd. (Shanghai, China), the microwave [G70F23CN2P-BM1 (SO)] used was from Glanze (Guangdong, China), the incubator (ZDP-A2160) was purchased from Hangzhou, Seoul Equipment Co., Ltd. (Hangzhou, China) and the microscope (IX73) was from Olympus Corp. (Tokyo, Japan).

Modeling. A total of 55 female and 5 male SD rats were randomly selected. The ratio of mated male to female rats at night was 2:1. Sperm were observed in the vaginal smear under the microscope and a vaginal plug was observed after $12 \mathrm{~h}$ (next day), which was considered to be the first day of pregnancy (2). Otherwise, after 7 days of separation between the male and female rats, the same process was repeated until they became pregnant. After 7 days of pregnancy, $16.6 \mathrm{mg} / \mathrm{kg}$ of mifepristone was administered at 8:00 a.m. and $0.1 \mathrm{mg} / \mathrm{kg}$ of misoprostol was administered at 6:00 p.m. causing the sac to discharge and vaginal bleeding. The rats were dissected 6 days post-treatment and no pregnancy tissue was identified in the uterus. Pathological examination showed a complete abortion. Thus, a rat model of complete termination (abortion) of early pregnancy was established (3).

Randomization. The rats undergoing abortion were numbered from 1 to 30 using a computerized random number table, and divided into the ultrasound irradiation or the sham irradiation group.

Treatment. From the first day of abortion, low-intensity focused ultrasound irradiation (sound intensity, $2 \mathrm{~W} / \mathrm{cm}^{2}$; frequency, $0.8 \mathrm{MHz}$ ) was applied to the abdominal area around the uterine tissue for $30 \mathrm{~min}$ daily for 5 consecutive days in the ultrasound irradiation group. Subsequent to shaving the abdomen, the anesthetized rats were placed in the supine position on the surgical table. The abdomen, bladder and the treatment head were coated with ultrasound coupling agent. Appropriate pressure was used to regulate the thickness of the bladder. The distance between the therapeutic transducer and abdominal skin was $1.5-2.5 \mathrm{~cm}$ (to ensure that the uterine tissue was within the ultrasonic focal region). The therapeutic transducer was rotated slowly for $30 \mathrm{~min}$.

The sham irradiation group was similarly treated without any power output in the therapeutic transducer (sound intensity, $0 \mathrm{~W} / \mathrm{cm}^{2}$; frequency, $0 \mathrm{MHz}$ ).

Detection indicators and methods. The measurement of ET-1 and NO was as follows: The proportion of tissue weight $(\mathrm{g})$ : Normal saline $(\mathrm{ml})=1: 9$. The tissues were centrifuged at 2,500 $\mathrm{r} / \mathrm{min}$ for $10 \mathrm{~min}$ and the supernatants were used to measure ET-1 and NO levels by ELISA and chemical testing.

The OXTR measurement was as follows: Uterine tissue samples were fixed in $4 \%$ paraformaldehyde, embedded in paraffin wax and sliced at of $4-40 \mu \mathrm{m}$. The slices were mounted
Table I. Effect of low-intensity ultrasound on ET-1 and NO levels of uterine tissue.

\begin{tabular}{lccc}
\hline Groups & No. & ET-1 $(\mathrm{ng} / \mathrm{l})$ & $\mathrm{NO}(\mu \mathrm{mol} / \mathrm{g}$ prot $)$ \\
\hline Ultrasound radiation & 15 & $98.83 \pm 27.72$ & $1.614 \pm 0.849$ \\
Sham radiation & 15 & $69.47 \pm 24.92$ & $2.231 \pm 1.735$ \\
$\mathrm{t} / \mathrm{Z}$ & & $\mathrm{t}=3.051$ & $\mathrm{Z}=-0.933$ \\
P-values & & $\mathrm{P}=0.005$ & $\mathrm{P}=0.367$ \\
\hline
\end{tabular}

ET-1, endothelin-1; NO, nitrogen monoxide.

on slides, dehydrated using alcohol washes of increasing concentrations (such as 50, 75, 90, 95 and 100\%), and cleared using xylene. OXTR was detected by immunohistochemistry.

Image analysis. Cells with yellow particles were considered to exhibit a positive expression of OXTR. A total of 5 high-power fields were randomly selected in each slice. The number of positive cells $<10 \%$ was recorded as $0 ; 10-30 \%$ as $1 ; 30-50 \%$ as $2 ; 50-70 \%$ as 3 ; and $>70 \%$ as 4 . Color intensity was also used for scoring as follows: Pale yellow was recorded as 1, yellow as 2 and brown as 3 . The two scores (0-7) were added to calculate the following: Negative $(0,-)$, weakly positive $(1-2,+)$, moderately positive $(3-4,++)$ and strongly positive $(5-7,+++)$.

Statistical analysis. The data were processed using SPSS for Windows Version 20.0 (IBM Corp., Armonk, NY, USA). Measurement data are described as mean \pm standard deviation, and categorical data as ratios or rates. When the measurement data showed normality and homogeneity of variance between the two groups, t-tests were used. Otherwise, the Wilcoxon rank-sum test was used. Categorical data were analyzed by Pearson's $\chi^{2}$ test. $\mathrm{P}<0.05$ was considered to indicate a statistically significant difference.

\section{Results}

Pathology. In the ultrasound irradiation group, the uterine muscle fibers were slender, the inner muscles were arranged in a ring and the outer muscles were longitudinal (Fig. 1A and B). In the sham irradiation group, the uterine muscle fibers showed mild hypertrophy and were haphazardly arranged. Additionally, the myometrium was thicker and the uterine cavity was larger compared to the ultrasound irradiation group (Fig. 1C and D).

ET-1 level in the uterine tissue. The ET-1 level in the uterine tissues was significantly higher in the ultrasound irradiation group compared to the sham irradiation group $(\mathrm{P}<0.05)$ (Table I).

NO level in the uterine tissue. The NO level in the uterine tissues was lower in the ultrasound irradiation group compared to the sham irradiation group $(\mathrm{P}>0.05)$ (Table I).

OXTR expression in the myometrium. Positive OXTR expression was observed in the myometrium of the two groups. 
Table II. OXTR expression in the myometrium in the two groups.

\begin{tabular}{lcccccc}
\hline Group & No. & $(-)$ & $(+)$ & $(++)$ & $(+++)$ & $(>++\%)$ \\
\hline Ultrasound radiation & 15 & 0 & 0 & 3 & 12 & 80.00 \\
Sham radiation & 15 & 0 & 2 & 7 & 6 & 40.00 \\
Total & 30 & 0 & 2 & 10 & 18 & \\
\hline
\end{tabular}

Signifcant difference of the strong positive expression rate between the two groups $\left(\chi^{2}=5.000, \mathrm{P}=0.025<0.05\right)$. OXTR, oxytocin receptor.

Table III. OXTR expression in the endometrium of the uterus in the two groups.

\begin{tabular}{lcccccc}
\hline Group & No. & $(-)$ & $(+)$ & $(++)$ & $(+++)$ & $(>++\%)$ \\
\hline Ultrasound radiation & 15 & 0 & 0 & 2 & 13 & 86.70 \\
Sham radiation & 15 & 0 & 2 & 7 & 6 & 40.00 \\
Total & 30 & 0 & 2 & 9 & 18 & \\
\hline
\end{tabular}

Signifcant difference of the strong positive expression rate between the two groups $\left(\chi^{2}=7.033, \mathrm{P}=0.008<0.05\right)$. OXTR, oxytocin receptor.

However, $80 \%$ uterine tissues in the ultrasound irradiation group showed strong positive expression, in contrast to only $40 \%$ in the sham irradiation group. The difference was statistically significant $(\mathrm{P}<0.05)$ (Table II) (Fig. 2A and C).

OXTR expression in the endometrium. Positive OXTR expression was observed in the endometrium of the two groups.
However, $86.7 \%$ uterine tissues in the ultrasound irradiation group showed strong positive expression, in contrast to only $40 \%$ in the sham irradiation group. The difference was statistically significant $(\mathrm{P}<0.05)$ (Table III) (Fig. 2B and D).

\section{Discussion}

Incomplete uterine involution frequently occurs postpartum, which seriously affects the physical and mental health of women. Its incidence has increased with the increase in cesarean section deliveries (4). Slow decline of uterine fundus prevents the uterus from returning to a normal size in puerperium, causes uterine displacement, increased lochia amount, duration, and even hemorrhage or infections in puerperium. Uterine smooth muscle contraction is necessary for uterine involution and $70-80 \%$ of postpartum hemorrhages are caused by uterine inertia (5).

ET-1 and NO are involved in the regulation of uterine contraction and relaxation (6). ET is the strongest and most persistent vasoconstrictor peptide known, and has four isoenzymeisomers:ET-1,ET-2,ET-3 and vasoactive intestinal peptide contraction. Uterine tissue mainly generates ET-1 (7), which promotes muscle contraction by Gq-phospholipase C-inositol 1,4,5-trisphosphate signal transduction pathways, protein kinase $\mathrm{C} \theta(\mathrm{PKC} \theta)$ and phospholipase $\mathrm{A} 2$ to activate the synthesis of arachidonic acid, PKC and sphingosine kinase, which in turn activate RhoA/Rho kinase (7-9). NO is an endothelium-derived relaxing factor, which activates the guanylyl cyclase (GC) to inhibit spontaneous uterine smooth muscle contraction $(10,11)$. GC is widely present in smooth muscle cells, and its activation increases the cyclic guanosine monophosphate (cGMP) level, which facilitates smooth muscle contraction and relaxation.
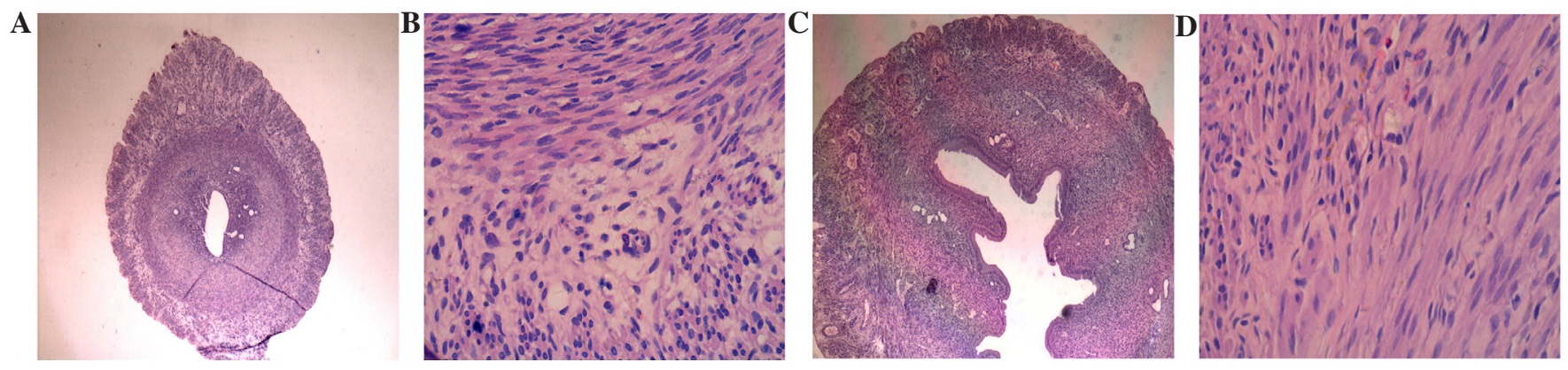

Figure 1. Hematoxylin and eosin staining of the (A and B) ultrasound and (C and D) sham irradiation groups. (A and C) Magnification, $\mathrm{x} 40$. (B and D) Magnification, $x 400$.
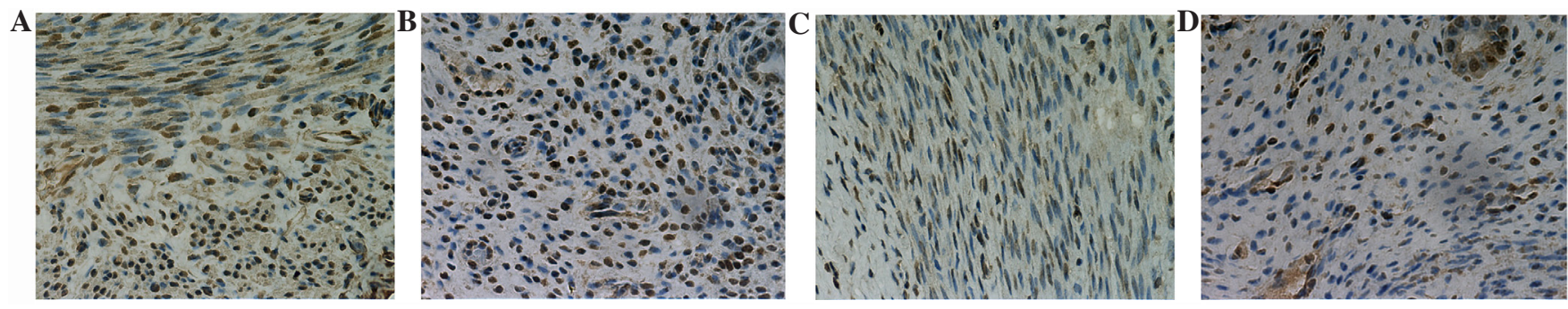

Figure 2. Immunohistochemistry showing a (A and B) strong and (C and D) weakly positive expression in the (A and C) myometrium and (B and D) endometrium. Magnification, $\mathrm{x} 400$. 
Oxytocin (OT) is a neuropeptide that is mainly produced in the supraoptic nuclei and paraventricular nuclei of the hypothalamus (12), and is released into the central nervous system and peripheral blood (13). It can stimulate uterine muscle contraction, promote milk secretion, and has a role in the functions of the breast, ovary, brain and uterus. OT exerts its biological effects by binding to the OXTR (14), which enhances uterine muscle contraction in rats, humans, rabbits and cows (15). Prolonged OT stimulation will desensitize OXTR in the uterine smooth muscle $(16,17)$. Therefore, increasing the expression of OXTR in postpartum uterine smooth muscle cells may be effective in managing incomplete uterine involution.

In 1978, Ter Haar et al (18) discovered that ultrasound $\left(3 \mathrm{MHz}, 2 \mathrm{~W} / \mathrm{cm}^{2}\right)$ can promote the contraction of uterine smooth muscle in pregnant mice, and enhance the frequency and amplitude of spontaneous uterine contraction. Zhang et al and Rui-Hong et al (19-21) reported that ultrasonic irradiation can promote the contraction of uterine smooth muscle in rats and humans, which were confirmed by in vivo studies. After ultrasonic (frequency, 0.8 MHz; sound intensity, $2 \mathrm{~W} / \mathrm{cm}^{2}$ ) irradiation for $10 \mathrm{~min}$, the frequency, amplitude, tension and contraction of the isolated uterus in rats increased significantly. These parameters were considered to be the most appropriate radiation intensity.

The present study used low-intensity focused ultrasound (frequency $=0.8 \mathrm{MHz}$, sound intensity $=2 \mathrm{~W} / \mathrm{cm}^{2}$ ) to irradiate the uterus following medical abortion in rats. In the ultrasound irradiation group, uterine muscle fibers were slender, inner muscles were arranged in a ring and the outer muscles were longitudinal. In the sham irradiation group, uterine muscle fibers showed mild hypertrophy and were haphazardly arranged. In addition, the myometrium was thicker and the uterine cavity was larger compared to the ultrasound irradiation group. These results suggested that low-intensity ultrasound can promote contraction of uterine muscle, thereby promoting uterine involution. Low-intensity ultrasound affected the levels of NO and ET-1 in aborted uterine tissue of rats. ET-1 levels in the uterine tissues were significantly higher in the ultrasound irradiation group compared to the sham irradiation group $(\mathrm{P}<0.05)$. However, $\mathrm{NO}$ levels in the uterine tissues were slightly less in the ultrasound compared to the sham irradiation group, but there was no statistical significance $(\mathrm{P}>0.05)$. Under normal circumstances, ET and NO exhibit negative feedback regulation and promote their synthesis by endothelin receptors, while NO uses cGMP to inhibit the production of ET. The two are synthesized and released in dynamic equilibrium, thereby maintaining a relatively stable ratio. Any imbalance results in a pathological state. These results suggest that low-intensity ultrasound altered the balance of ET-1 and NO to reach a new homeostasis, which was more suitable for the contraction of the uterus.

Zhang et al (21) showed that ultrasound can significantly increase OT-induced contraction of uterine smooth muscle. The frequency and amplitude of contraction, and uterine activity were significantly enhanced. Therefore, whether ultrasound promotes uterine involution was investigated by increasing the expression of OXTR in the uterine tissue. OT combined with OXTR, which is expressed on myometrium cells, causes direct contraction of uterine smooth muscle. OXTR can stimulate epithelial cells to release prostaglandins, and promote contraction by a paracrine mechanism (22). Therefore, the present study analyzed OXTR expression in the endometrium and myometrium of the 2 groups, and identified that the strong positive expression of OXTR was significantly higher in the ultrasound irradiation group compared to the sham irradiation group $(\mathrm{P}<0.05)$ thereby suggesting that increased expression of OXTR is an important mechanism by which low-intensity ultrasound promotes uterine involution.

Low-intensity focused ultrasound (sound intensity, $2 \mathrm{~W} / \mathrm{cm}^{2}$; frequency, $0.8 \mathrm{MHz}$ ) could promote uterine involution in rats that had undergone complete abortion, and thus promote the recovery of tissue morphology. The underlying mechanisms include an increase in ET-1 levels, modifying the balance of ET-1 and NO, and the expression of OXTR in the uterine myometrium and endometrium.

\section{Acknowledgements}

The present study was supported by the National Natural Science Fund by the Chinese National Science Foundation (grant nos. 81127901 and 31000435), the National Basic Research Program of China (grant nos. 2011CB707900 and 2012CB722402), the National Twelfth Five-year Science and Technology Support Program (grant no. 2011BAI141301) and Science and Technology Project affiliated to the Education Department of Chongqing Municipality (grant nos. KJ110325 and KJ130329). The authors thank Mrs. Chongyan Li, Mr. Jie Xu, Mrs. Chengdan Ran and Mrs. Cuiping Wang for their contribution to this study.

\section{References}

1. Menetrey J, Kasemkijwattana C, Fu FH, Moreland MS and Huard J: Suturing versus immobilization of a muscle laceration. A morphological and functional study in a mouse model. Am J Sports Med 27: 222-229, 1999.

2. Li Y, Shao L, Li S, Deng Z and Zhu C: Experimental observation on the Pessaries for the identification of pregnant rat model. Chinese Journal of Laboratory Animal Science 1: 6, 2002.

3. You Z, Zeng J, You H, Zha Y, Deng H and Li D: Effect of gynecology hemostasis acesodyne granule on the expression of estrogen-progestin and its receptor in rats model of medical abortion. Chinese Journal of Information on Traditional Chinese Medicine 17: 19-21, 2010.

4. Wu Z, Zeng Y and Zhu L: Analysis of 238 cases postpartum subinvolution of uterus. The Modern Medicine 11: 41-42, 2011.

5. Heinemann U: Basic mechanisms of partial epilepsies. Curr Opin Neurol 17: 155-159, 2004

6. Yang J, Yang T and Cui X: Effect of Sheng Hua Tang to the level of NO and ET-1 in abortion rat model. Journal of Liaoning Traditional Chinese Medicine 11: 2002-2003, 2009.

7. Dallot E, Pouchelet M, Gouhier N, Cabrol D, Ferré F and Breuiller-Fouché M: Contraction of cultured human uterine smooth muscle cells after stimulation with endothelin-1. Biol Reprod 68: 937-942, 2003.

8. Di Liberto G, Dallot E, Eude-Le Parco I, Cabrol D, Ferré F and Breuiller-Fouché M: A critical role for PKC zeta in endothelin-1-induced uterine contractions at the end of pregnancy. Am J Physiol Cell Physiol 285: C599-C607, 2003.

9. Tanfin Z, Leiber D, Robin P, Oyeniran C and Breuiller-Fouché M: Endothelin-1: Physiological and pathological roles in myometrium. Int J Biochem Cell Biol 43: 299-302, 2011.

10. Buhimschi I, Yallampalli C, Dong Y-L and Garfield RE: Involvement of a nitric oxide-cyclic guanosine monophosphate pathway in control of human uterine contractility during pregnancy. Am J Obstet Gynecol 172: 1577-1584, 1995.

11. Anggård EE: Endogenous and exogenous nitrates. Acta Anaesthesiol Scand Suppl 97: 7-10, 1992. 
12. Robinson KJ, Hazon N, Lonergan M and Pomeroy PP: Validation of an enzyme-linked immunoassay (ELISA) for plasma oxy tocin in a novel mammal species reveals potential errors induced by sampling procedure. J Neurosci Methods 226: 73-79, 2014.

13. Neumann ID and Landgraf R: Balance of brain oxytocin and vasopressin: Implications for anxiety, depression, and social behaviors. Trends Neurosci 35: 649-659, 2012.

14. Liu Q, Qian DM, Liu QL and Gao H: Research progress of oxytocin and its analogues. Chin J Pharm Anal 31: 609-613, 2011.

15. Murata T, Narita K and Ichimaru T: Rat uterine oxytocin receptor and estrogen receptor $\alpha$ and $\beta$ mRNA levels are regulated by estrogen through multiple estrogen receptors. J Reprod Dev 60 55-61, 2014.

16. Jia R and Liu X: Effects of oxytocin and prostaglandins on oxytocin receptor expression of human myometrial smooth muscle cell. Chin J Biochem Pharmaceuties 30: 379-382, 2009.

17. Magalhaes JK, Carvalho JC, Parkes RK, Kingdom J, Li Y and Balki M: Oxytocin pretreatment decreases oxytocin-induced myometrial contractions in pregnant rats in a concentration-dependent but not time-dependent manner. Reprod Sci 16: 501-508, 2009 .
18. Ter Haar G, Dyson M and Talbert D: Ultrasonically induced contractions in mouse uterine smooth muscle in vivo. Ultrasonics 16: 275-276, 1978.

19. Zhang Y, Sun JC, Chang SF, Wang ZB, Wang GZ and Liu YM Dose effect of low intensity ultrasound on uterine smooth muscle in rats. Acta Academiae Medicinae Militaris Tertiae 16: 1566-1568, 2007.

20. Rui-Hong L, Jiangchuan S and Shufang C: The effect of low intensity ultrasound on the contraction of uterine smooth muscle in rats in vivo. Journal of Chongqing Medical University 6: 16 , 2008.

21. Zhang Y, Sun JC, Chang SF and Wang ZB: A comparative study: Low intensity ultrasound and oxytocin on the contraction of uterine smooth muscle in rats. Chinese Journal of Medical Imaging Technology 2: 2, 2008.

22. Romero R, Sibai BM, Sanchez-Ramos L, Valenzuela GJ, Veille JC, Tabor B, Perry KG, Varner M, Goodwin TM, Lane R, et al: An oxytocin receptor antagonist (atosiban) in the treatment of preterm labor: A randomized, double-blind, placebo-controlled trial with tocolytic rescue. Am J Obstet Gynecol 182: 1173-1183, 2000. 